\title{
Positive lifestyle behaviour changes among Canadian men: Findings from the HAT TRICK program
}

\begin{abstract}
Purpose: To estimate program effectiveness regarding physical activity (PA), diet, and social connectedness as part of a feasibility study.

Design: Pre-post quasi-experimental.

Setting: HAT TRICK was delivered in collaboration with a Canadian semi-professional ice hockey team and offered at the arena where they trained and played games.

Participants: Participants $(\mathrm{N}=62)$ at baseline were overweight $\left(\mathrm{BMI}>25 \mathrm{~kg} / \mathrm{m}^{2}\right)$ and inactive ( $<150$ minutes of MVPA/week) men age 35+ years.

Intervention: Gender-sensitised 12-week intervention for men targeting PA, healthy eating and social connectedness.

Method: Baseline, post-intervention (12 weeks) and 9-month follow-up self-report and accelerometer data were collected. Multi-level modelling assessed growth trajectories of outcome measures across time.
\end{abstract}

Results: Accelerometer measured weekly/min. of moderate PA showed significant linear trends (95\%CI: 42.9 - 175.3) from baseline (147.0 \pm 104.6$), 12$-week $(237.7 \pm 135.5)$ and 9month follow-up (204.89 \pm 137.7$)$ qualified with a quadratic trend. Self-reported weekly/min of moderate and vigorous PA showed significant linear trends (95\%CI: 94.1, 264.1; 95\%CI: $35.1,109.6)$ from baseline $(52.6 \pm 83.8,22.42 \pm 44.9), 12$ week $(160.1 \pm 157.4,66.6 \pm 74.4)$ and 9-month follow-up $(118.6 \pm 104.6,52.2 \pm 59.2)$ qualified with quadratic trends. DINE measured 
fat score rating showed linear trends over time $(95 \% \mathrm{CI}-14.24,-6.8)$, qualified with a quadratic trend. DINE fibre score and social connectedness showed no trends.

Conclusion: Findings yield valuable information about the implementation of gendersensitised lifestyle interventions for men and demonstrate the importance of male-specific strategies for reaching and engaging overweight, physically inactive men.

Key Words: men's health, health behavior, physical activity, diet, social connectedness

Trial Registration: This trial is registered with clinicaltrials.gov (XXXXXXX) and the International Clinical Trials Registry Platform (XXXXXXXXX). 


\section{Purpose}

Regular physical activity, consuming a healthy diet, and engaging in practices to promote psychological well-being can reduce the likelihood of developing chronic diseases including cardiovascular disease, type 2 diabetes, cancer and depression ${ }^{1}$. Despite the physical and mental benefits associated with these healthy lifestyle behaviors, up to $83 \%$ of Canadian men do not meet the recommended physical activity guidelines ${ }^{2}$, and over $62 \%$ of Canadian men engage in unhealthy dietary behaviors, including high fat intake and inadequate fruit and vegetable consumption ${ }^{3}$. Moreover, there is mounting evidence that engaging in poor lifestyle behaviors, weight gain, and mental illness are closely linked as a result of shared pathophysiological pathways and processes ${ }^{4}$.

A fundamental reason why many men avoid taking preventive actions for their health, is that health promotion initiatives rarely address the influence of masculinities ${ }^{5}$. Specifically, some men say they are reticent to access or attend preventive health services and programs because they perceive this as threatening to masculine ideals of strength, selfreliance and independence ${ }^{5,6}$. Moreover, men's estrangement from health promotion initiatives (i.e., programs to enhance physical activity, healthy eating and activities promoting psychological well-being) can reflect the lack of appeal that these initiatives hold for them and/or their irrelevance to their masculine identities ${ }^{6}$. As a consequence, poor engagement and program uptake are common ${ }^{7}$. Thus, when designing and implementing health promoting initiatives for men, it is crucial to take into account prevailing constructions of masculinities ${ }^{8}$.

Integrated gender-sensitized approaches that combine physical activity and healthy eating and promote aspects of psychological well-being may be effective for engaging men in health promoting lifestyle behaviors. For example, activities that enhance a sense of belonging, foster teamwork, camaraderie and social connectedness using male-specific 
engagement strategies (e.g., group based format with male facilitators and role models, meeting in a 'male-friendly' setting such as a sports arena, using humor and 'banter' during group sessions) have consistently been reported as crucial elements to program uptake, engagement and sustainability ${ }^{9-11}$. Further, researchers have highlighted the value of social contact and group-based activities in locations where men often congregate and feel comfortable for improving the social-emotional and physical health of men ${ }^{9}$.

The HAT TRICK program ${ }^{12}$ is a gender-sensitized, group-based intervention focused on supporting physical activity, healthy eating and social connectedness among overweight and inactive men, and delivered in collaboration with a major junior (i.e., level preceding professional) Canadian ice hockey team. It was specifically created to address the masculine constructs associated with engaging and retaining men in health promoting initiatives and this is reflected in the design, content, setting, and delivery of the program. For example, the content utilized hockey-related themes to frame health-related topics and emphasized strategies that appeal to prevailing masculinities, such as mastery and group/team dynamics 9,13; the program was delivered at the hockey arena where our partner team trained and played home games (i.e., 'male-friendly' environment) ${ }^{8,9,11}$; and all messaging was delivered using frank and familiar communication styles, utilizing humor and 'banter' where possible, as these have been reported as important components to engaging men in health promotion programs ${ }^{13,14}$.

The overarching aim of the HAT TRICK evaluation was to determine intervention feasibility and acceptability, and secondly, to estimate effectiveness in terms of physical activity, diet, and psychological well-being, including social connectedness. Further details concerning the feasibility and acceptability of the gender-sensitized components of HAT TRICK are reported elsewhere ${ }^{10}$. This article reports on the secondary aim and provides an 
exploratory analysis of the effectiveness of HAT TRICK on physical activity, diet, and social connectedness.

\section{Methods}

\section{Study Design}

HAT TRICK's intervention design and methodological protocol have been detailed elsewhere ${ }^{12}$. In brief, this exploratory study utilized a pre-post, quasi-experimental design. Baseline, post-intervention (12 weeks) and 9 month follow-up measures were completed between December 2016 and January 2018. All participants provided informed consent prior to baseline assessments. Ethics approval was obtained from the XXXXX (\#H1600736) and this trial is registered with clinicaltrials.gov (XXXXXXX) and the International Clinical Trials Registry Platform (XXXXXXX).

\section{Sample}

A variety of recruitment methods were employed, including: local news media (e.g., print and digital newspapers, radio broadcasts); social media (e.g., Facebook, Twitter); poster advertisements at community centers, ice hockey arenas, pubs and bars, hardware and automotive commercial entities, and face-to-face flyer handouts at the ice hockey teams home games. A project specific website was developed as an additional recruitment strategy, and to provide information about the project (i.e., eligibility criteria, information about what the program included). Lastly, participants who had completed initial HAT TRICK sessions assisted with the recruitment of subsequent groups by word of mouth. Interested participants were encouraged to contact the research team by telephone in order to determine eligibility.

To be eligible, men had to be over the age of 35 years; residing in the region; selfreport the accumulation of less than 150 minutes of physical activity per week and a body mass index (BMI) of over $25 \mathrm{~kg} / \mathrm{m}^{2}$ with a pant waist size of 38 " or greater; and if required, 
medical clearance from a physician after screening positive using the Physical Activity Readiness Questionnaire (PAR-Q+) ${ }^{15}$. Because group session delivery was limited to a maximum of 20 men, eligible individuals were accepted on a 'first come first served' basis with additional individuals being placed on a waitlist for the next available session.

\section{HAT TRICK Intervention}

HAT TRICK ${ }^{12}$ is a 12-week group-based intervention focused on three specific components including physical activity, diet, and social connectedness. Each 90-minute weekly session comprised of targeted health education (e.g., how to accumulate physical activity throughout the day, understanding macronutrients, managing symptoms of depression, anxiety, and stress), while simultaneously promoting enjoyment and increased social connectedness through an interactive and informal style of learning. For instance, to enhance social connectedness, facilitators fostered a sense of teamwork and camaraderie among the men through group activities and competition. Part of each 90-minute session was allocated to progressive physical activity (i.e., duration and intensity of physical activity increased each week) using a variety of 'men-friendly' activities such as road/floor hockey, strength and resistance training, and walking. The intervention is theory-guided, drawing on multiple constructs from the Social Cognitive Theory ${ }^{16}$ and Self-Determination Theory ${ }^{17}$ and on health models and theories which incorporate gender and socially constructed masculinities ${ }^{18}$ as an important social determinant.

All participants were provided with a wearable device (i.e., Fitbit Charge2 ${ }^{\mathrm{TM}}$ ) and encouraged to use this to self-monitor progress towards graduated increases in daily physical activity. Men also received an individual resource manual called the 'Playbook' which provided tips and resources for healthy living, information about behavior change techniques (e.g., goal setting, social support, self-monitoring) and a log to track physical activity and dietary behaviors. In addition, relevant HAT TRICK team personnel and community health 
professionals were invited as guest presenters to some sessions. For instance, the hockey team's fitness trainer delivered a 'boot camp' type exercise session focused on building muscular strength and a local registered dietician presented and discussed how to choose healthier food options and tips for controlling portion sizes.

\section{Measures}

All measures, expect for demographic information, were assessed at baseline, postintervention (12 weeks) and 9 month follow-up.

\section{Participant characteristics}

Demographic information, including date of birth, ethnic background, level of education, marital status, co-morbidities (heart disease, hypertension, high cholesterol, diabetes, cancer, stroke, arthritis, mental health problems respiratory disease), main physical activity, occupation and household income were self-reported. Ethnic background categories were taken from the Canadian Census questionnaire and were provided in list form for participants to select (White, South Asian (e.g., East Indian, Pakistani, Sri Lankan), Chinese, Black, Filipino, Arab, Latin American, Southeast Asian (e.g., Vietnamese, Cambodian, Laotian, Thai), West Asian (e.g., Iranian, Afghan), Korean, Japanese, Other: specify ${ }^{19}$. Anthropometric and physiological variables, including height $(\mathrm{cm})$, weight $(\mathrm{kg})$, waist circumference $(\mathrm{cm})$, blood pressure $(\mathrm{mmHg})$, and heart rate (beats/min) were assessed by a research team member, trained to a standard protocol.

Objectively measured physical activity

An ActiGraph GT3X accelerometer (ActiGraph, Pensacola, Florida, USA) was used to objectively measure physical activity (mild, moderate and vigorous) and step counts. It was worn on the participants' hip during waking hours over seven days (at each time assessment period), except during bathing or other water-related activities. Participant data were downloaded in 60-second epochs, and established cut-off points were used to calculate 
daily minutes of moderate (2691-6166 counts/min) and vigorous ( $>6167$ counts/min) physical activity ${ }^{20}$. Only those with total daily wear times of at least 600 minutes on four or more days were included in the analyses.

\section{Self-reported physical activity}

Physical activity was also assessed by self-report using the valid and reliable modified version of the Godin Leisure Time Exercise Questionnaire-GLTEQ ${ }^{21}$. The GLTEQ classifies physical activity/exercise into three intensity subgroups: vigorous, moderate, and light activity. Participants were asked to report the frequency, intensity and duration (minutes) of their weekly physical activity.

\section{Dietary behavior}

Dietary behaviors were assessed using the validated Dietary Instrument for Nutrition Education (DINE) ${ }^{22}$. This 19-item food frequency questionnaire assesses dietary intake of total fat and dietary fiber over a 7 day period. Composite scores were calculated in accordance with the DINE scoring protocol ${ }^{22}$, with higher scores indicating greater consumption of total fat and dietary fiber.

\section{Social Connectedness}

Social support was assessed using the valid and reliable abbreviated Duke Social Support Index (DSSI-11) ${ }^{23}$. This 11-item index contains two subscales, social interaction and satisfaction with social support, evaluated on a four-point Likert scale. The total score for the DSSI-11 ranges from 10 to 30 with higher scores indicating stronger perception of social support $^{23}$.

\section{Analysis}

Demographic data were first analyzed descriptively. Secondly, dependent variable linear and quadratic trends were analyzed between baseline, post-program and 9-month follow-up using multi-level modelling (MLM) and intention to treat analyses to estimate 
effects ${ }^{24}$. MLM was used for its ability to account for random effects. Initial modelling began with linear and quadratic components and a random effect for the intercept estimated on the linear term, to assess the shape of growth trajectories and to assess whether the means of the accelerometer, GLTEQ, DINE, social connectedness and BMI measures differed across time. Covariance estimates were assessed to determine if a random intercept was appropriate for the model. Models were re-run if covariance estimates suggested no between participant variability. Differing covariance structures were assessed in an attempt to define the best model indicated by the smallest Akiaike's Information Criterion (AIC). Analyses were run using SPSS version 22.

\section{Results}

\section{Demographics}

Sixty-two male participants with a mean age of $50.98(\mathrm{SD}=10.09$, range $=35-77)$ years completed self-report measures at baseline, 58 completed post program measures and 54 completed 9 month follow-up measures. Participant flow is outlined in Figure 1.The majority of participants had a post-secondary degree, worked full time, and were married. Full demographic measures can be seen in Table 1.

Multilevel modeling showed BMI having no significant linear $(p=.066)$ or quadratic trends $(p=.465)$.

\section{Accelerometer measured physical activity}

Sixty participants wore accelerometers at baseline, 56 post-program and 52 at 9 month follow up. At baseline one participant had insufficient wear time, as did four at postprogram and eight at follow up measures. Average daily wear times for those with sufficient wear was $808.46(203.55) \mathrm{min}$ at baseline, 842.79 (225.73) $\mathrm{min}$ at post program and 756.37 (143.05) min at follow-up. 
Initial multilevel models showed moderate physical activity having linear growth trends over time $(p<.001)$, qualified with a quadratic trend $(p<.001)$. Total weekly step counts also showed linear growth trends over time $(p=.014)$, qualified by a quadratic trend $(p=.032)$. Mild physical activity and vigorous physical activity did not show linear or quadratic trends. Means and standard deviations across time points are shown in Table 2. Estimates of fixed effects and 95\% confidence intervals are shown in Table 3. 


\section{Self-report measures}

Self-reported vigorous physical activity showed a linear growth trend over time $(p<$

$3.001)$, qualified with a quadratic trend $(p=.004)$. Mild physical activity showed no marked

4 trends over time. Self-reported moderate physical activity showed linear growth trends over

5 time $(p<.001)$, qualified with a quadratic trend $(p=.001)$.

6 DINE measured fat score rating showed decreasing linear trends over time $(p<.001)$,

7 qualified with a quadratic trend $(p=.001)$. The DINE fiber score showed no marked trends. trends over time.

All self-reported means and standard deviations are shown in Table 2. Estimates of

fixed effects and 95\% confidence intervals are shown in Table 3. All models had random intercepts for participants unless otherwise stated in Table 3.

\section{Discussion}

HAT TRICK was designed as a new, gender-sensitized intervention aimed at attracting, engaging and retaining a sub-group of men in healthier lifestyle behavior change.

17 Results from this exploratory study showed increases in both objectively measured (i.e., accelerometry) and self-reported moderate PA. Participants' self-reported vigorous PA increased over time, although similar increases in vigorous PA were not seen in the accelerometer data. However, accelerometer data did show an increase in step-count. Concerning diet, results indicated a decrease the DINE fat score, from scores representing moderate fat intake at baseline to scores indicating low fat intake at both follow-up time points. The DINE fiber score, which is based on fruit and vegetable consumption, did not change. There were also no indication of changes in our measure of social connectedness. BMI showed no notable significant differences. Taken together, these results provide 
1 promising evidence that HAT TRICK is likely to be an effective strategy for engaging more

2 men in positive and sustainable lifestyle behavior changes.

The sustained improvements in moderate physical activity, assessed objectively and

4 subjectively, aligns with previous gender-sensitized interventions ${ }^{25}$ and may further support

5 program acceptability claims and the notion that men may more readily engage in health

6 behavior change programs which focus on activities that promote feelings of strength and

7 mastery, such as preferred forms of physical activity ${ }^{11,26}$. Previous work has also suggested

8 that when working towards achieving a healthy weight, programs which forefront physical

9 activity are particularly attractive to men because this plays to masculine identities. For

10 example, men's enjoyment of physical activity and motivation to try new forms of physical

activity has been linked to the nostalgia for playing team sports as a younger man, and the masculine appeal of expert physical training to improve athletic performance, as well as the camaraderie and allyship that can develop among men working (and playing) together to achieve shared goals ${ }^{10,11,25}$. In contrast, too strong a focus on engagement in healthy eating can challenge normative masculinities and gendered food roles ${ }^{26}$. Our results also indicated an increase in self-reported vigorous physical activity, but

17 this was not supported by the accelerometer data. Although over-reporting of physical activity is recognized as a common issue of self-report instruments ${ }^{27}$, in this study these differences may also be related to men's interpretation of the increase in physical activity participation (over the 12 week intervention) and the exposure to new, more intense activities

21 (e.g., 'bootcamp' style session) as working harder than they actually were. However, this

22 finding should not be disregarded because it does suggest that the men have become more aware of their physical activity as a result of taking part in the program and hence may have started to build greater self-efficacy and confidence surrounding their engagement and 25 participation in physical activity. 
2 on the use of a documented 'gold standard' objective measure of physical activity (i.e.,

3 accelerometer) and findings from our HAT TRICK formative evaluation ${ }^{10}$, we confidently

4 speculate that the design of our intervention may have played a major role in these promising

5 results. A key focus of our intervention was the use of simple, real and frank

6 messaging/communication about physical activity and healthy eating, as this has been

7 reported as a crucial strategy for engaging and sustaining men in preventive health behaviors

$8{ }^{13,14}$. For instance, the program regularly highlighted the impact that including small,

9 incremental changes in physical activity could have on overall health benefit (e.g., accumulating small $3 \times 10$ minute bouts of activity throughout the day; taking the stairs at least once every day instead of an escalator). To foster engagement and recognize masculine ideals of competitiveness ${ }^{11,13,14}$, we set up weekly personal step challenges and gave the men Fitbits so that they could self-monitor their daily steps (and other activity), and this internal competition may have served well to motivate the men to do more ${ }^{11,14}$. Together, these elements proved to be beneficial in increasing steps and overall PA levels.

A substantial reduction in the DINE fat score was observed in the current study but no corresponding change in the fiber score. The reduction in the fat score of approximately 12 units suggests a reduction in fat intake to $<30 \mathrm{~g} /$ day or $\sim 270$ calories ${ }^{28}$. The reduction in fat intake can be attributed to the messaging related to the macronutrient content of foods in the HAT TRICK program (e.g., encouragement to choose lower fat versions of commonly consumed foods, and if consuming a fatty type food, to just eat less). The results for dietary fiber were less encouraging in that total fiber intake may have actually gone down. However, the scores from baseline to 36-weeks follow-up fell within a 'medium' fiber score which equates to $21-30 \mathrm{~g} / \mathrm{day}^{28}$. Current recommendations for men aged 19-50 in Canada is to reach a fiber intake $38 \mathrm{~g} / \mathrm{day}^{28}$, and so this result suggests a different messaging approach 
1 may be required to reach that target. The small reduction in fiber intake may be related to a

2 reduction in total intake (i.e., eating less) but because the DINE assessment does not assess

3 total intake (i.e., calories) we can only speculate on our interpretation for this result.

4 Nevertheless, the changes observed for the fat score are encouraging given that supporting

5 men to make healthy changes in their diet has been shown to be a challenge ${ }^{29}$.

6 A lack of any notable change in social support over time was surprising. Recent

7 research has challenged assumptions that men are disinterested in establishing close

8 relationships with others ${ }^{30}$, and suggested that men commonly establish social relationships

9 with other men through sports and physical activities - a practice that is aligned with

10 hegemonic masculinities and avoids social taboos in building social connections with other

11 men ${ }^{30}$. While HAT TRICK included opportunities for men to meet as a group in weekly

12 interactive sessions involving physical activity, it did not appear to be sufficient to influence

13 men's perceptions of social support. The HAT TRICK program may benefit from providing

14 opportunities for men to explore and challenge gender-related expectations about men's

15 social relationships, and demonstrate practices to support positive social connections and

16 relationships among men ${ }^{10}$.

\section{Strengths, limitations, and future directions}

There are strengths and limitations to this research that must be considered when interpreting the findings. HAT TRICK was specifically designed to address a need to engage more men in sustainable healthy behavior change. In this regard, the program addresses a need for evidence-based health promotion interventions that consider the influence of prevailing cultures of masculinities and how these may promote or curtail particular behaviors. This research is also strengthened by the use of objectively (i.e., accelerometry) measured physical activity. While some previous studies in men's health have incorporated the use of objectively measured steps (i.e., pedometers) for evaluation purposes, 
1 accelerometers provide additional valuable information such as the intensity of activity, a

2 variable that cannot be assessed by pedometer ${ }^{31}$. It should be noted that accelerometers can

3 have difficulties assessing exercises in a fixed position.

The exploratory nature of this study as reflected in the modest sample size and lack of

5 a control group limit any conclusions regarding the overall effectiveness of the program.

6 Future testing of HAT TRICK, through a fully powered randomized control trial, is needed to

7 claim effectiveness. Further, despite efforts to recruit a diverse sample of men, participants

8 were predominantly white, well educated, and with higher incomes. Thoughtful consideration

9 should be given to reaching less well-resourced men whose access to such programs is

10 inhibited by health inequities. Further, the HATTRICK model does show promise for

11 transferability across other sport settings ${ }^{25,32}$ however particular adaptions are required to

12 ensure acceptability. For example, the 'look and feel' of the program and the tools and

resources utilized for program delivery, will need to reflect the particular sport. It should also

14 be noted that not all men will relate to prevailing cultures of masculinities, such as sport, and

15 the HAT TRICK program is only one possible avenue of addressing the health and wellbeing

16 of certain types of men.

"SO WHAT?" (100 - 150 words $)$

\section{What is already known on this topic?}

20 Up to $83 \%$ of Canadian men do not meet the recommended physical activity guidelines and over $62 \%$ of Canadian men engage in unhealthy dietary behaviors, including high fat intake and inadequate fruit and vegetable consumption. Many men are reticent to access or attend preventive health services/programs because they perceive this as threatening to masculine

24 ideals of strength, self-reliance and independence. 


\section{$1 \quad$ What does this article add?}

2 HAT TRICK was designed as a new, gender-sensitized intervention aimed at attracting,

3 engaging and retaining a sub-group of men in healthier lifestyle behavior change and shows

4 promising impacts on men's physical activity levels and healthy eating habits.

5

6 What are the implications for health promotion practice or research?

7 This paper highlights a practical way to engage more men in positive healthy eating, active

8 living and social behaviours.

10 Funding

This research was supported by the Canadian Cancer Society (grant no XXXXXX).

XXXXXX is supported by an Australian Government Research Training Program

Scholarship. The content is solely the responsibility of the authors and does not necessarily represent the official views of the Canadian Cancer Society. The study sponsor was not involved in the design of the study; the collection, analysis, and interpretation of data; writing the report; or the decision to submit the report for publication.

\section{Compliance with Ethical Standards}

\section{Authors' statement of conflict of interest}

Authors XXXXXXXXXXX declare that they have no conflict of interest.

\section{Research involving human participants}

All procedures performed in this study were in accordance with the ethical standards of the institutional and/or national research ethics committee and with the 1964 Helsinki

24 declaration and its later amendments or comparable ethical standards. 
Informed consent was obtained from all individual participants included in the study.

\section{References}

4 1. World Health Organisation. Global health risks: Mortality and the burden of disease

2. Colley RC, Garriguet D, Janssen I, Craig CL, Clarke J, Tremblay MS. Physical activity of Canadian adults: accelerometer results from the 2007 to 2009 Canadian Health Measures Survey. Health Rep. 2011;22(1):7-14.

9 3. Flannigan RK, Oliffe JL, McCreary DR, et al. Composite health behaviour classifier as the basis for targeted interventions and global comparisons in men's health. Can Urol Assoc J. 2019;13(4):125-132.

4. Chauvet-Gelinier JC, Roussot A, Cottenet J, et al. Depression and obesity, data from a national administrative database study: Geographic evidence for an epidemiological overlap. PLoS One. 2019;14(1):e0210507.

5. Courtenay WH. Constructions of masculinity and their influence on men's well-being: a theory of gender and health. Soc Sci Med. 2000;50(10):1385-1401.

6. Sloan C, Gough B, Conner M. Healthy masculinities? How ostensibly healthy men talk about lifestyle, health and gender. Psychol Health. 2010;25(7):783-803.

7. Pagoto SL, Schneider KL, Oleski JL, Luciani JM, Bodenlos JS, Whited MC. Male inclusion in randomized controlled trials of lifestyle weight loss interventions. Obesity (Silver Spring). 2012;20(6):1234-1239.

8. Oliffe JL, Rossnagel E, Bottorff JL, Chambers SK, Caperchione CM, Rice SM. Community-based men's health promotion programs: Eight lessons learnt and their Caveats. Health Promot Int. 2019. 
19 . Bottorff JL, Seaton CL, Johnson ST, et al. An updated review of interventions that include promotion of physical activity for adult men. Sports Med. 2015;45(6):775800.

10. Sharp P, Bottorff JL, Hunt K, et al. Men's perspectives of a gender-sensitized health promotion program targeting healthy eating, active living, and social connectedness. Am J Mens Health. 2018;12(6):2157-2166.

11. Hunt K, McCann C, Gray CM, Mutrie N, Wyke S. 'You've got to walk before you run': Positive evaluations of a walking program as part of a gender-sensitized, weightmanagement program delivered to men through professional football clubs. Health Psychology. 2013;32(1):57.

12. Caperchione CM, Bottorff JL, Oliffe JL, et al. The HAT TRICK programme for improving physical activity, healthy eating and connectedness among overweight, inactive men: study protocol of a pragmatic feasibility trial. BMJ Open. 2017;7(9):e016940.

13. Gray CM, Hunt K, Mutrie N, et al. Football Fans in Training: the development and optimization of an intervention delivered through professional sports clubs to help men lose weight, become more active and adopt healthier eating habits. BMC public health. 2013;13(1):232.

14. Morgan PJ, Collins CE, Plotnikoff RC, et al. Efficacy of a workplace-based weight loss program for overweight male shift workers: the Workplace POWER (Preventing Obesity Without Eating like a Rabbit) randomized controlled trial. Prev Med. 2011;52(5):317-325.

15. Canadian Society for Exercise Physiology. Physical Activity and Readiness Questionnaire for everyone: PAR-Q+. Canadian Society for Exercise Physiology: CSEP. 
http://www.csep.ca/cmfiles/publications/parq/parqplussept2011version all.pdf.

Published 2012. Accessed 21, Feb 2017.

16. Bandura A. Social foundation of thought and action: A social cognitive theory. Englewood Cliffs, NJ: Prentice-Hall; 1986.

17. Teixeira PJ, Carraca EV, Markland D, Silva MN, Ryan RM. Exercise, physical activity, and self-determination theory: a systematic review. Int J Behav Nutr Phys Act. 2012;9:78.

18. Creighton G, Oliffe JL. Theorising masculinities and men's health: A brief history with a view to practice. Health Soc Rev. 2010;19(4):409-418.

19. Canada S. 2021 Census: 2A-L. Statistics Canada. https://www.statcan.gc.ca/eng/statistical-programs/instrument/3901_Q2 V6. Published 2020. Accessed 07-2020, 2020.

20. Troiano RP, Berrigan D, Dodd KW, Masse LC, Tilert T, McDowell M. Physical activity in the United States measured by accelerometer. Med Sci Sports Exerc. 2008;40(1):181-188.

21. Godin G, Jobin J, Bouillon J. Assessment of leisure time exercise behavior by selfreport: a concurrent validity study. Can J Public Health. 1986;77(5):359-362.

22. Roe L, Strong C, Whiteside C, Neil A, Mant D. Dietary intervention in primary care: validity of the DINE method for diet assessment. Fam Pract. 1994;11(4):375-381.

23. Koenig HG, Westlund RE, George LK, Hughes DC, Blazer DG, Hybels C. Abbreviating the Duke Social Support Index for use in chronically ill elderly individuals. Psychosomatics. 1993;34(1):61-69.

24. Casals M, Girabent-Farres M, Carrasco JL. Methodological quality and reporting of generalized linear mixed models in clinical medicine (2000-2012): a systematic review. PLoS One. 2014;9(11):e112653. 
1 25. Wyke S, Hunt K, Gray CM, et al. Football Fans in Training (FFIT): a randomised controlled trial of a gender-sensitised weight loss and healthy living programme for men-end of study report. Public Health Res. 2015;3(2):1-129.

26. Archibald D, Douglas F, Hoddinott P, et al. A qualitative evidence synthesis on the management of male obesity. BMJ Open. 2015;5(10):e008372.

27. Limb ES, Ahmad S, Cook DG, et al. Measuring change in trials of physical activity interventions: a comparison of self-report questionnaire and accelerometry within the PACE-UP trial. Int J Behav Nutr Phys Act. 2019;16(1):10.

28. Dieticians of Canada. Food sources of fibre Canada: Health Canada-Food and Nutrition;2016.

29. Taylor PJ, Kolt GS, Vandelanotte C, et al. A review of the nature and effectiveness of nutrition interventions in adult males--a guide for intervention strategies. Int J Behav Nutr Phys Act. 2013;10:13.

30. Oliffe JL, Rice S, Kelly MT, et al. A mixed methods study of the health-related masculine values among young Canadian men. Psychol Men Masculin. 2019;20:310323.

31. Kelly LA, McMillan DG, Anderson A, Fippinger M, Fillerup G, Rider J. Validity of actigraphs uniaxial and triaxial accelerometers for assessment of physical activity in adults in laboratory conditions. BMC Med Phys. 2013;13(1):5.

32. Maddison R, Hargreaves EA, Wyke S, et al. Rugby Fans in Training New Zealand (RUFIT-NZ): a pilot randomized controlled trial of a healthy lifestyle program for overweight men delivered through professional rugby clubs in New Zealand. BMC Public Health. 2019;19(1):166. 
1 33. Canada LaA. Metis Nation. Government of Canada. https://www.bac-

2 lac.gc.ca/eng/discover/aboriginal-heritage/metis/Pages/introduction.aspx. Published

3 2020. Accessed July 2020, 2020.

4

5 
1 Figure 1 Participant Flow Diagram

2

3

4

5

6

7

8

9

10

11

12

13

14

15

16

17

18

19

20

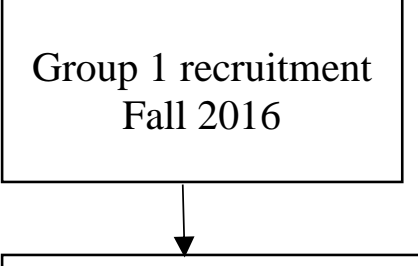

Group 2 recruitment

Winter 2017

Eligibility determined, PAR-Q+ and informed consent completed prior to each baseline assessment period

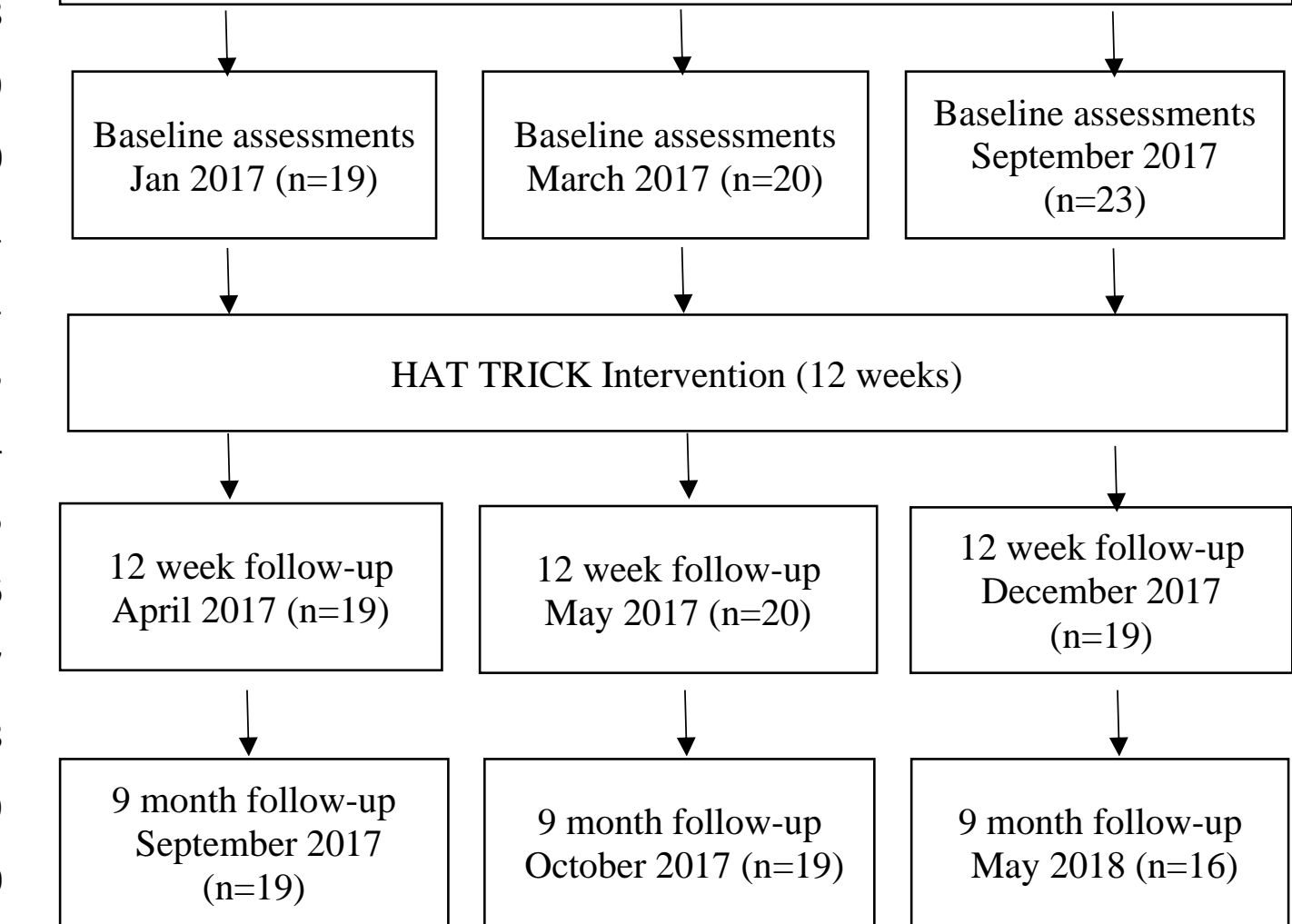

21

22 
1 Table 1 Demographic Measures

\begin{tabular}{|c|c|c|}
\hline Measure & Frequency $(n=62)$ & Percentage \\
\hline \multicolumn{3}{|l|}{ Highest Level of Education } \\
\hline Some high school or less & 1 & $1.6 \%$ \\
\hline High school diploma & 13 & $20.9 \%$ \\
\hline College or technical diploma / University degree & 48 & $77.4 \%$ \\
\hline \multicolumn{3}{|l|}{ Household Income Before Taxes } \\
\hline$\$ 25,000-\$ 49,999$ & 6 & $9.7 \%$ \\
\hline$\$ 50,000-\$ 99,999$ & 26 & $41.9 \%$ \\
\hline$\$ 100,000$ or more & 30 & $48.4 \%$ \\
\hline \multicolumn{3}{|l|}{ Main Activity } \\
\hline Full time work & 50 & $80.6 \%$ \\
\hline Part time work & 1 & $1.6 \%$ \\
\hline Caring for family/managing household/retired & 11 & $17.7 \%$ \\
\hline \multicolumn{3}{|l|}{ Marital Status } \\
\hline Married/domestic partnership & 54 & $87.1 \%$ \\
\hline Divorced/separated & 5 & $8.1 \%$ \\
\hline Single/never married/widowed & 3 & $4.8 \%$ \\
\hline \multicolumn{3}{|l|}{ Ethnic Background } \\
\hline White & 57 & $91.9 \%$ \\
\hline Metis $^{1}$ & 1 & $1.6 \%$ \\
\hline Latin & 1 & $1.6 \%$ \\
\hline South Asian & 1 & $1.6 \%$ \\
\hline West Asian & 1 & $1.6 \%$ \\
\hline Other & 1 & $1 . \%$ \\
\hline
\end{tabular}




\begin{tabular}{|c|c|c|}
\hline \multicolumn{3}{|l|}{ BMI Category } \\
\hline Overweight $\left(25-<30 \mathrm{~kg} / \mathrm{m}^{2}\right)$ & 5 & $8.1 \%$ \\
\hline Obese Class $1\left(30-<35 \mathrm{~kg} / \mathrm{m}^{2}\right)$ & 21 & $33.9 \%$ \\
\hline Obese Class $2\left(35-<40 \mathrm{~kg} / \mathrm{m}^{2}\right)$ & 25 & $40.3 \%$ \\
\hline Obese Class $3\left(40+\mathrm{kg} / \mathrm{m}^{2}\right)$ & 11 & $17.7 \%$ \\
\hline \multicolumn{3}{|l|}{ Co-morbidities } \\
\hline None & 19 & $30.6 \%$ \\
\hline One & 21 & $33.9 \%$ \\
\hline Two or more & 22 & $35.5 \%$ \\
\hline
\end{tabular}


Table 2 Means and Standard Deviations Across Time Points with MLM linear and quadratic 95\% Confidence Interval.

\begin{tabular}{|c|c|c|c|c|c|}
\hline & $\begin{array}{c}\text { Baseline } \\
\text { Mean (SD) }\end{array}$ & $\begin{array}{l}\text { 12-weeks } \\
\text { Mean (SD) }\end{array}$ & $\begin{array}{c}\text { 36-weeks Follow-up } \\
\text { Mean (SD) }\end{array}$ & Linear $95 \% \mathrm{CI}$ & Quadratic 95\% CI \\
\hline \multicolumn{6}{|c|}{ Weekly Physical Activity (PA) } \\
\hline \multicolumn{6}{|c|}{ GLTEQ (minutes/week) } \\
\hline Mild PA & $157.58(202.61)$ & $206.25(172.51)$ & $236.87(276.26)$ & $-53.70,162.17$ & $-55.53,43.47$ \\
\hline Moderate PA & $52.67(83.77)$ & $160.09(157.41)$ & $118.58(104.57)$ & $94.10,264.10$ & $-116.30,-32.08$ \\
\hline Vigorous PA & $22.42(44.91)$ & $66.57(74.42)$ & $52.21(59.23)$ & $35.07,109.66$ & $-46.88,-9.27$ \\
\hline \multicolumn{6}{|c|}{ Accelerometer (minutes/week) } \\
\hline Mild PA & $1743.76(408.44)$ & $1759.06(598.55)$ & $1696.38(501.45)$ & $-194.96,260.7$ & $-131.79,94.14$ \\
\hline Moderate PA & $147.00(104.63)$ & $237.70(135.47)$ & $204.89(137.73)$ & 42.98, 175.27 & $-76.79,-0.84$ \\
\hline Vigorous PA & $2.00(5.17)$ & $3.15(6.38)$ & $1.89(4.21)$ & $-0.72,6.15$ & $-3.08,0.41$ \\
\hline Step Count & $\begin{array}{r}39,175.44 \\
(14,747.59)\end{array}$ & $\begin{array}{r}45,507.56 \\
(19,160.22)\end{array}$ & $\begin{array}{r}42,145.20 \\
(20,544.97)\end{array}$ & 1464.86, 20916.13 & $-9754.46,207.81$ \\
\hline \multicolumn{6}{|l|}{ DINE } \\
\hline Fat Score & $36.60(14.57)$ & $24.77(11.02)$ & $24.15(12.69)$ & $-14.24,-6.80$ & $1.36,4.84$ \\
\hline
\end{tabular}




\begin{tabular}{|l|r|r|r|r|r|}
\hline Fibre Score & $24.32(10.46)$ & $23.68(12.04)$ & $20.95(14.03)$ & $-4.74,5.53$ & $-3.47,1.39$ \\
\hline Duke Social Support Index & $26.21(3.08)$ & $26.5(4.09)$ & $26.96(3.52)$ & $-1.39,1.48$ & $-0.61,0.81$ \\
\hline Social Support & $8.27(1.40)$ & $8.48(1.51)$ & $8.59(1.25)$ & $-0.38,0.90$ & $-0.37,0.25$ \\
\hline Social Interaction & $17.93(2.39)$ & $18.02(3.21)$ & $18.37(2.85)$ & $-1.37,0.95$ & $-0.41,0.73$ \\
\hline Satisfaction with Social & & & & \\
Support & & & & \\
\hline BMI & & & & \\
\hline BMI & $36.19(6.02)$ & $35.78(6.30)$ & $35.19(6.67)$ & $-1.50,0.05$ & $-0.23,0.50$ \\
\hline
\end{tabular}

PA=Physical activity; GLTEQ=Godin Leisure Time Exercise Questionnaire; SD=Standard deviations; CI=Confidence Interval

Bold text indicated statistical significance 
Table 3. Multilevel Modelling Estimates of Fixed Effects and Akiaike's Information Criterion

\begin{tabular}{|c|c|c|c|c|}
\hline \multirow[t]{2}{*}{ Dependent Variable } & \multirow[t]{2}{*}{$t(\mathrm{df})$} & \multicolumn{2}{|c|}{ Estimates of Fixed Effects } & \multirow{2}{*}{$\begin{array}{c}\text { Akiaike's Information } \\
\text { Criterion }\end{array}$} \\
\hline & & $\begin{array}{l}\text { Estimate } \\
\text { (Std. Error) }\end{array}$ & $95 \% \mathrm{CI}$ & \\
\hline \multicolumn{5}{|c|}{ Physical Activity (PA)-GLTEQ } \\
\hline Mild PA & & & & 2284.56 \\
\hline Intercept & $t(61)=6.12$ & $157.58(25.73)$ & $106.13,209.03$ & \\
\hline Linear & $t(78.98)=1.00$ & $54.23(54.22)$ & $-53.70,162.17$ & \\
\hline Quadratic & $t(80.11)=-0.24$ & $-6.05(24.86)$ & $-55.53,43.47$ & \\
\hline Moderate PA & & & & 2081.14 \\
\hline Intercept & $t(105.94)=3.54$ & $54.91(15.50)$ & $24.18,85.65$ & \\
\hline Linear & $t(62.82)=4.21$ & $179.10(45.53)$ & $94.10,264.10$ & \\
\hline Quadratic & $t(59.46)=-3.52$ & $-74.19(21.05)$ & $-116.30,-32.08$ & \\
\hline Vigorous PA & & & & 1841.58 \\
\hline Intercept & $t(61.00)=3.93$ & $22.42(5.7)$ & 11.01, 33.82 & \\
\hline
\end{tabular}




\begin{tabular}{|c|c|c|c|c|}
\hline $\begin{array}{l}\text { Linear } \\
\text { Quadratic }\end{array}$ & $\begin{array}{l}t(54.87)=3.89 \\
t(55.92)=8.94)\end{array}$ & $\begin{array}{l}73.37(18.61) \\
-28.07(9.39)\end{array}$ & $\begin{array}{r}35.07,109.66 \\
-46.88,-9.27\end{array}$ & \\
\hline \multicolumn{5}{|c|}{ Physical Activity (PA)-Accelerometer } \\
\hline Mild PA $\mathbf{A}^{\mathrm{F}}$ & & & & 2117.75 \\
\hline Intercept & $t(66.26)=26.56$ & $1742.71(65.61)$ & $1611.71,18.73 .70$ & \\
\hline Linear & $t(50.41)=0.29$ & $32.87(113.46)$ & $-194.96,260.71$ & \\
\hline Quadratic & $t(56.14)=46.47$ & $-18.82(56.14)$ & $-131.79,94.14$ & \\
\hline Moderate PA $^{\mathrm{F}}$ & & & & 1785.45 \\
\hline Intercept & $t(56.07)=12.03$ & $167.57(13.93)$ & $139.66,195.48$ & \\
\hline Linear & $t(52.95)=3.31$ & $109.13(32.98)$ & $42.98,175.27$ & \\
\hline Quadratic & $t(51.34)=-2.05$ & $-38.82(18.92)$ & $-76.79,-0.84$ & \\
\hline Vigorous PA & & & & 851.40 \\
\hline Intercept & $t(55.51)=2.76$ & $1.91(0.69)$ & $0.52,3.30$ & \\
\hline Linear & $t(47.95)=1.59$ & $2.72(1.71)$ & $-0.72,6.15$ & \\
\hline Quadratic & $t(50.06)=-1.53$ & $-1.33(0.87)$ & $-3.08,0.41$ & \\
\hline
\end{tabular}




\begin{tabular}{|c|c|c|c|c|}
\hline Step Counts $^{\mathrm{F}}$ & & & & 3142.87 \\
\hline Intercept & $t(55.28)=19.63$ & $38763.44(1974.22)$ & $34807.46,42719.41$ & \\
\hline Linear & $t(52.84)=2.31$ & $11190.50(4848.55)$ & 1464.86, 20916.13 & \\
\hline Quadratic & $t(50.26)=-1.92$ & $-4773.32(2480.27)$ & $-9754.46,207.81$ & \\
\hline \multicolumn{5}{|c|}{ Duke Social Support Index } \\
\hline Social Support & & & & 866.38 \\
\hline Intercept & $t(61.00)=66.91$ & $26.21(0.39)$ & $25.43,26.99$ & \\
\hline Linear & $t(54.72)=0.06$ & $0.47(0.71)$ & $-1.39,1.48$ & \\
\hline Quadratic & $t(56.06)=0.29$ & $0.10(0.35)$ & $-0.61,0.81$ & \\
\hline Social Interaction & & & & 579.65 \\
\hline Intercept & $t(69.68)=44.17$ & $8.27(0.19)$ & $7.90,8.64$ & \\
\hline Linear & $t(63.95)=0.80$ & $0.25(0.32)$ & $-0.38,0.90$ & \\
\hline Quadratic & $t(55.41)=-0.38$ & $-0.06(0.15)$ & $-0.37,0.25$ & \\
\hline \multicolumn{5}{|c|}{ Satisfaction with Social Support } \\
\hline Intercept & & & & 787.57 \\
\hline Linear & $t(61.00)=59.32)$ & $17.94(0.30)$ & $17.33,18.54$ & \\
\hline
\end{tabular}




\begin{tabular}{|c|c|c|c|c|}
\hline Quadratic & $\begin{aligned} t(55.68) & =-0.36 \\
t(56.43) & =0.58\end{aligned}$ & $\begin{array}{r}-0.21(0.58) \\
0.17(0.28)\end{array}$ & $\begin{array}{l}-1.37,0.95 \\
-0.41,0.73\end{array}$ & \\
\hline \multicolumn{5}{|l|}{ DINE } \\
\hline $\begin{array}{l}\text { Fat Score } \\
\text { Intercept } \\
\text { Linear } \\
\text { Quadratic }\end{array}$ & $\begin{aligned} t(68.82) & =21.90 \\
t(79.30) & =-5.63 \\
t(61.00) & =3.57\end{aligned}$ & $\begin{array}{r}25.84(1.18) \\
-10.52(1.87) \\
3.10(0.87)\end{array}$ & $\begin{array}{r}23.48,28.19 \\
-14.24,-6.80 \\
1.36,4.84\end{array}$ & 1307.56 \\
\hline $\begin{array}{l}\text { Fibre Score } \\
\text { Intercept } \\
\text { Linear } \\
\text { Quadratic }\end{array}$ & $\begin{array}{r}t(73.23)=18.18 \\
t(75.86)=0.15 \\
t(61)=-0.85\end{array}$ & $\begin{array}{r}24.32(1.34) \\
0.39(2.58) \\
-1.04(1.22)\end{array}$ & $\begin{array}{r}\text { 21.66, 26.99 } \\
-4.74,5.53 \\
-3.47,1.39\end{array}$ & 1407.85 \\
\hline \multicolumn{5}{|l|}{ BMI } \\
\hline $\begin{array}{l}\text { BMI } \\
\text { Intercept } \\
\text { Linear } \\
\text { Quadratic }\end{array}$ & $\begin{array}{r}t(64.68)=45.45 \\
t(73.16)=-1.87 \\
t(58.34)=0.74\end{array}$ & $\begin{array}{c}36.19(0.80) \\
-0.73(0.39) \\
0.13(0.18)\end{array}$ & $\begin{array}{r}\mathbf{3 4 . 6 0}, \mathbf{3 7 . 7 8} \\
-1.50,0.05 \\
-0.23,0.50\end{array}$ & 846.61 \\
\hline
\end{tabular}


F = Fixed intercept; PA=Physical activity; GLTEQ=Godin Leisure Time Exercise Questionnaire; DINE=Dietary Instrument of Nutrition Education

Bold text indicated statistical significance 